\title{
Estudo de uma nova técnica para criação cirúrgica de um defeito semelhante a meningomielocele em fetos de coelhos ${ }^{1}$
}

\author{
Denise A. L. Pedreira ${ }^{2}$ \\ Paulo R. Valente ${ }^{3}$ \\ Rogério C. Abou-Jamra ${ }^{3}$ \\ Cláudio L. Pelarigo ${ }^{4}$ \\ Lilian M. Silva ${ }^{5}$ \\ Saul Goldenberg ${ }^{6}$
}

\begin{abstract}
Pedreira DAL, Valente P, Abou-Jamra RC, Pelarigo CL, Silva LM, Goldenberg S. Estudo de uma nova técnica para criação cirúrgica do defeito semelhante a meningomielocele em fetos de coelho. Acta Cir Bras [serial online] 2003 Mar-Abr;18(2). Disponível em URL: http://www.scielo.br/acb.

RESUMO - Objetivo: Avaliar a sobrevida fetal usando uma nova técnica cirúrgica para criação de um defeito semelhante a meningomielocele em fetos de coelho. Métodos: Foram utilizadas seis coelhas da linhagem Nova Zelândia que tiveram um defeito espinhal criado em seus fetos no $23^{\circ}$ dia de gestação. O defeito foi criado em 19 fetos e a tentativa de correção foi feita em 15 casos (grupo I), 4 fetos permaneceram sem correção (grupo II). No $30^{\circ}$ dia de gestação, os fetos foram submetidos a avaliação anátomo-patológica. Resultados: No $30^{\circ}$ dia a meningomielocele estava presente em todos os fetos sobreviventes. A sobrevida total foi de 73,68\% (14/19), 11 fetos sobreviveram no grupo I, e 3 no grupo II. Conclusão: A técnica modificada apresenta sobrevida fetal satisfatória, no modelo experimental de criação de defeito espinhal em fetos.
\end{abstract}

DESCRITORES - Cirurgia fetal. Meningomielocele. Disrafismo espinhal. Coelho. Pré-natal. Modelos animais de doenças.

1. Trabalho realizado no Laboratório de Cirurgia Experimental do Instituto Dante Pazzanese de Cardiologia - São Paulo.

2. Doutora em Medicina pela Universidade de São Paulo Coordenadora dos Projetos de Cirurgia Fetal Experimental do Laboratório de Cirurgia Experimental do Instituto Dante Pazzanese de Cardiologia. Coordenadora do Serviço de Medicina Fetal do Hospital Israelita Albert Einstein.

3. Médico Veterinário Responsável pelo Laboratório de Cirurgia Experimental do Instituto Dante Pazzanese de Cardiologia.

4. Médico Veterinário Estagiário do Laboratório de Cirurgia Experimental do Instituto Dante Pazzanese de Cardiologia.

5. Médica do Departamento de Anatomia Patológica do Instituto Dante Pazzanese de Cardiologia.

6. Chefe do Laboratório de Cirurgia Experimental do Instituto Dante Pazzanese de Cardiologia Titular do Departamento de Cirurgia da UNIFESP-EPM. Pesquisador categoria 1A do CNPq. 


\section{Introdução}

A meningomielocele é uma doença devastadora, podendo levar a retardo mental, deficiência motora e incontinência urinária/fecal, dependendo de sua gravidade. O tratamento pós-natal oferece poucas possibilidades de melhora aos recém-nascidos afetados e o tratamento pré-natal parece ser a escolha mais razoável. Quanto mais cedo a medula puder ser recoberta, menor o grau de lesão mecânica e química a que ela será submetida e menor o grau de herniação posterior do cerebelo (mal formação de Arbnold -Chiari tipo II $)^{1,2,3}$.

A possibilidade de melhora do quadro neurológico com a correção cirúrgica pré-natal da meningomielocele, foi primeiramente demonstrada em modelo animal ${ }^{4,5,6}$ e a correção vem sendo recentemente realizada em humanos ${ }^{1,2}$. Como resultado, o desenvolvimento de novas técnicas para a correção do defeito tornou-se um campo de pesquisa novo e importante.

A ovelha tem sido o animal de escolha para estudar a correção antenatal do defeito, provavelmente por seu feto ter tamanho semelhante ao feto humano ${ }^{7,8,9,10}$. Embora esta seja uma vantagem, o período gestacional longo e o número pequeno de fetos presente em cada gestação, pode ser desfavorável, especialmente para o desenvolvimento de novas técnicas e teste de novos materiais. Para este propósito, acreditamos que o coelho seja o melhor modelo animal por causa de seu período gestacional curto e maior número de fetos (6-8 por gestação). Estas características facilitam a constituição de um grupo controle, permitindo rápidas mudanças nas técnicas e/ou materiais, se necessário. Além disso, o coelho tem um manejo mais simples quando comparado à ovelha.

Apesar disso, só ultimamente, o coelho foi estabelecido como um modelo para a criação cirúrgica do defeito ${ }^{11,12}$, ainda não tendo sido estabelecido como um modelo para sua correção. Com este objetivo em mente, demos início a um protocolo para estudar a correção da meningomielocele, usando para sua criação inicialmente a técnica descrita por Calvano e col. ${ }^{11}$, subseqüentemente modificada por Housley e col. ${ }^{12}$. Durante nosso estudo piloto, acabamos por modificar estas técnicas e pretendemos descrever estas modificações no presente estudo.

\section{Métodos}

Seis coelhas Nova Zelândia brancas grávidas adquiridas de um biotério comercial externo, que chegaram ao laboratório sete dias antes do ato operatório para aclimatação. Elas foram alojadas em gaiolas individuais, em um ambiente calmo com sucção de ar contínua, sendo submetidas a ciclos naturais de luz do dia. A alimentação (fornecida pelo mesmo biotério) e água foram permitidas até 30 minutos antes da intervenção cirurgica, realizada no $23^{\circ}$ dia de gestação. A medicação pré-anestésica foi constituída de cloridrato de ketamina $20 \mathrm{mg} / \mathrm{Kg}$ e acepromazina $1 \mathrm{mg} / \mathrm{Kg}$, a metade da dose foi administrada por via intramuscular, 20 minutos antes da operação e antes da tricotomia. O animal era, então, conduzido à sala operatória, colocado deitado de costas com seus membros contidos, em cima de um colchão aquecido. Depois de obter um acesso venoso na lateral da orelha, a outra meia dose de pré-medicação era administrada, seguida de cefazolin endovenoso $25 \mathrm{mg} / \mathrm{Kg}$, usado para profilaxia antibiótica. Os eletrodos de monitorização cardíaca eram colocados e o acesso venoso era mantido por infusão contínua de solução salina. Halotano 1\%-2\% foi administrado por meio de uma máscara de ventilação modificada e o animal manteve movimentos respiratórios espontâneos. A medroxiprogesterona era também administrada na dose de $4,5 \mathrm{mg}$ por via intra-muscular para obter relaxamento uterino.

Após a colocação dos campos cirúrgicos, era realizada laparotomia mediana, sendo o útero exposto para contagem do número inicial de fetos viáveis. Só o feto a ser operado permanecia exposto, enquanto o resto era devolvido à cavidade abdominal. Usando a cauda fetal como ponto de referência através da palpação externa, duas suturas (seda 4-0) eram aplicadas no miométrio para expor, após a incisão uterina, a região da espinha lombo-sacra do feto. Ambas as suturas incluíam as membranas; a inclusão acidental do feto na sutura foi evitada colocando-se dois dedos ao lado do útero mantendo o feto afastado da região de sutura (Figura 1A). 

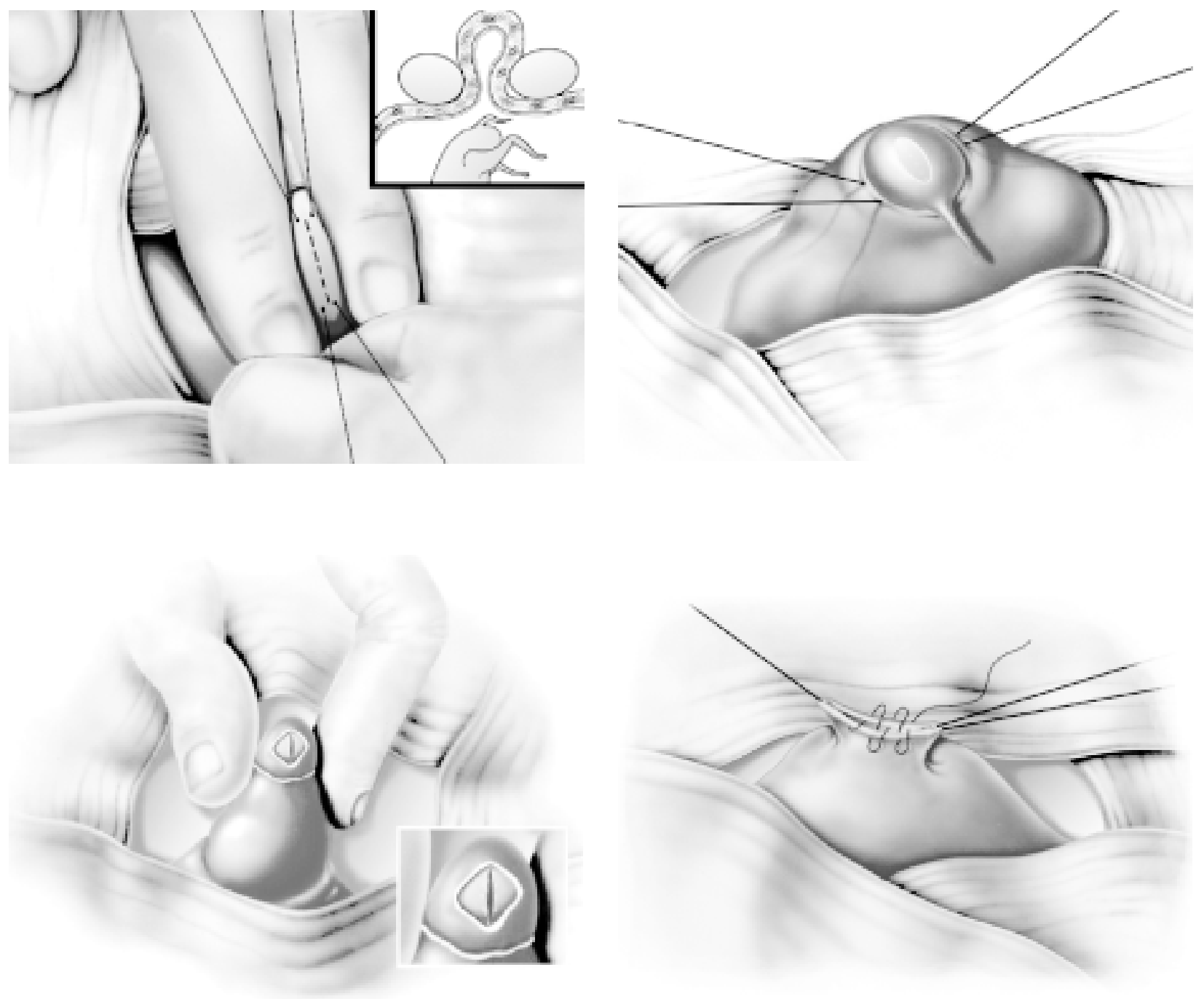

FIGURA 1 - Desenho esquemático da técnica cirúrgica usada para criação da meningomielocele em fetos de coelho. (A) Duas suturas de reparo foram aplicadas contemplando as membranas fetais (detalhe), precedendo a abertura do miométrio. (B) Apenas a cauda fetal é exposta para manter o feto posicionado; é removida de uma área de pele, expondo os processos espinhosos (pontos brancos). (C) Incisão sobre o processo espinhoso sendo feita. Observar a abertura do canal espinhal e conseqüente exposição da medula. Notar a pressão aplicada à inserção dos membros posteriores do feto que auxilia na exposição da medula. (D) Sutura invaginante aplicada para o fechamento do miométrio, incluindo as membranas fetais

Foi executada a incisão uterina usando o eletrocautério. A membrana corial e a amniótica eram abertas usando tesoura fina. O feto era manipulado de forma a permitir que apenas o rabo e a coluna lombosacra permanecessem fora da cavidade uterina (Figura 1B). Usando-se lupa com ampliação de 4x (Heine, Alemanha) e um foco de luz halógena (Heine, Alemanha), a pele sobre a espinha era, então, retirada (aproximadamente $0,5 \mathrm{~cm}^{2}$ ) e uma pequena incisão do arco vertebral posterior era executada no nível da inserção das patas traseiras, contemplando quatro a cinco vértebras, com profundidade suficiente para incluir a dura mater. Esta incisão foi executada enquanto os membros posteriores do feto eram comprimidos (no nível de suas inserções na pélvis fetal), levando a medula a "pular fora" do canal medular quando a dura-máter era aberta, permitindo visibilização da artéria espinhal (Figura 1C).

No máximo, quatro fetos foram operados em cada fêmea. A correção imediata foi tentada em um grupo (grupo I) usando duas técnicas diferentes que foram utilizadas em seqüência. A primeira técnica usada (A) consistiu na aplicação de uma película de celulose (Biofill, Fibrocel-Paraná, Brasil) diretamente sobre a área onde a pele havia sido previamente excisada; o tamanho aproximado da película de celulose era $5 \mathrm{~mm}$ maior da área previamente retirada, sendo que as bordas da película ficavam sobre a pele normal. A segunda técnica 
aplicada (B) consistiu na dissecção de aproximadamente $5 \mathrm{~mm}$ da pele, ao redor do defeito, abaixo da derme e a celulose foi posicionada abaixo da pele normal, cobrindo completamente o defeito.

Em alguns fetos (grupo II), após a realização do defeito nenhuma correção foi executada e eles eram reconduzidos à cavidade uterina (grupo controle). Uma sutura contínua invaginante foi usada para o fechamento de útero (incluindo as membranas) e $5 \mathrm{ml}$ de solução salina morna foram injetados antes da sutura ser amarrada (Figura 1D). Após o fechamento por planos, os animais voltavam às gaiolas para a recuperação, comida e água eram oferecidas $A d$ Libitum. A atividade dos animais e o sangramento vaginal eram monitorados. Com 30 dias de gestação as fêmeas eram submetidas a eutanásia, injetando-se 0,5$1,0 \mathrm{ml} / \mathrm{Kg}$ de T 61 (Hoechst Roussel Veterinário, São Paulo - Brasil) intratorácico. A seguir, os fetos eram retirados por laparotomia e também submetidos a eutanásia por injeção de T 61 intratorácica. Os fetos operados eram pesados, fotografados e fixados em formol $10 \%$. Seções transversais da espinha fetal (coloração Hematoxilina e eosina) eram analisadas em ambos os grupos.

O defeito foi criado em 19 fetos e a correção foi tentada em 15 casos (grupo I). Foram operados 3-4 fetos por coelha. A técnica (A) foi usada em oito fetos e a técnica (B) em sete. A correção não foi realizada em quatro fetos que constituíram o grupo II.

$\mathrm{O}$ projeto foi aprovado pelo comitê ético de pesquisa animal local.

\section{Resultados}

$\mathrm{Na}$ criação do defeito não aconteceram mortes maternas durante ou depois do procedimento.

No $30^{\circ}$ dia, 11 entre 15 fetos do grupo I tinham sobrevivido e, três entre quatro, estavam vivos no grupo II (Tabela 1).

TABELA 1 - Taxa de sobrevida de fetos de coelho submetidos a criação cirúrgica da meningomielocele no $23^{\circ}$ dia de gestação (grupo II), comparados com fetos em que foi tentada a correção (grupo I).

\begin{tabular}{l|c|c|c}
\hline Grupo & Operados (n) & Sobrevivência (n) & Total (\%) \\
\hline Grupo I (submetido a correção) & 15 & 11 & 73,3 \\
\hline Grupo II (controle) & 4 & 3 & 75,0 \\
\hline Total & 19 & 14 & 73,7 \\
\hline
\end{tabular}

A sobrevida geral foi $73,68 \%(14 / 19)$. No $30^{\circ}$ dia, o defeito estava presente em todos os fetos sobreviventes em ambos os grupos, já que as técnicas utilizadas não levaram à correção do defeito criado (Figura 2A detalhe).

Microscopicamente a lesão criada em todos fetos

(A)

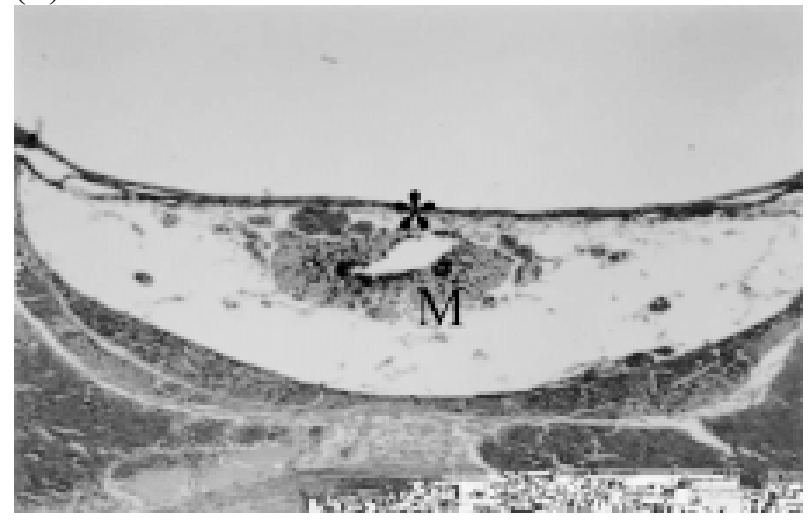

operados era semelhante às lesões presentes em fetos humanos afetados. A pele estava ausente e a medula exposta, enquanto uma fusão da pia e dura-máter pôde ser observada (Figura 2A). O achatamento da espinha, normalmente presente em casos humanos, pode ser visibilizado em alguns casos (Figura $2 \mathrm{~B}$ )

(B)

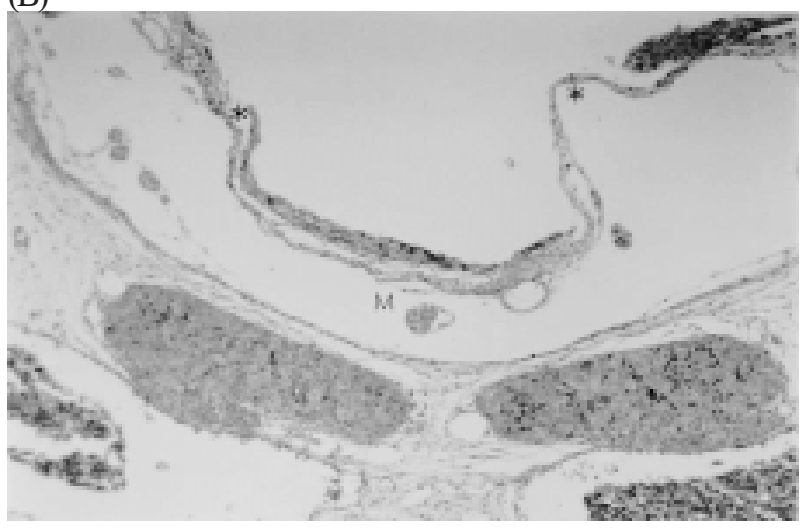

FIGURA 2 - Aspecto microscópico (coloração hematoxilina/eosina) da meningomielocele no $30^{\circ}$ dia de gestação. (A) Notar a fusão da pia e dura-máter $\left({ }^{*}\right)$ e o achatamento da medula $(\mathrm{M})$ presentes em alguns casos $(\mathrm{B})$. No detalhe, o aspecto macroscópico da lesão pode ser visto. 


\section{Discussão}

Existem, até o presente, apenas dois estudos, do mesmo grupo de autores, descrevendo a criação de um defeito semelhante à meningomielocele em fetos de coelho. Ambos relatam uma mortalidade fetal que varia de $40 \%{ }^{12}$ a $50 \%{ }^{11}$, enquanto em nossa série encontramos uma taxa de $26 \%$. Embora estes números não permitam avaliar se esta diferença seria estatísticamente significativa, nosso estudo é o primeiro a oferecer uma técnica alternativa válida para a produção do defeito, possivelmente mais segura que as técnicas anteriormente descritas. Quando comparamos nossos dados com estes outros estudos, devemos levar em conta a idade gestacional em que foi realizada a cirurgia fetal, pois esperamos observar uma maior mortalidade quanto menor for a idade gestacional na realização do procedimento $^{13}$. Ambos os estudos ${ }^{11,12}$ tiveram o procedimento realizado entre 22-23, quase na mesma idade gestacional em que os nossos casos foram operados (23 dias). Portanto, neste aspecto, os estudos podem ser comparados.

Comparando nossa técnica com os estudos anteriores, quanto ao acesso/posicionamento fetal, podese observar que Calvano e col. ${ }^{11}$ exteriorizava as patas traseiras até o nível da caixa torácica inferior para ter acesso à espinha, enquanto Housley ${ }^{12}$ tornava a espinha lombar e sacra acessível por meio de uma incisão uterina, mantendo o feto dentro do útero. Durante a fase piloto do nosso estudo, usando a técnica de Housley's ${ }^{12}$, uma das dificuldades foi manter a coluna fetal no lugar. Sendo que a manipulação fetal era muito grande para manter a espinha no lugar certo, especialmente durante a incisão do arco vertebral posterior, quando o feto tinha a tendência de "descer" em direção a cavidade

\section{(A)}

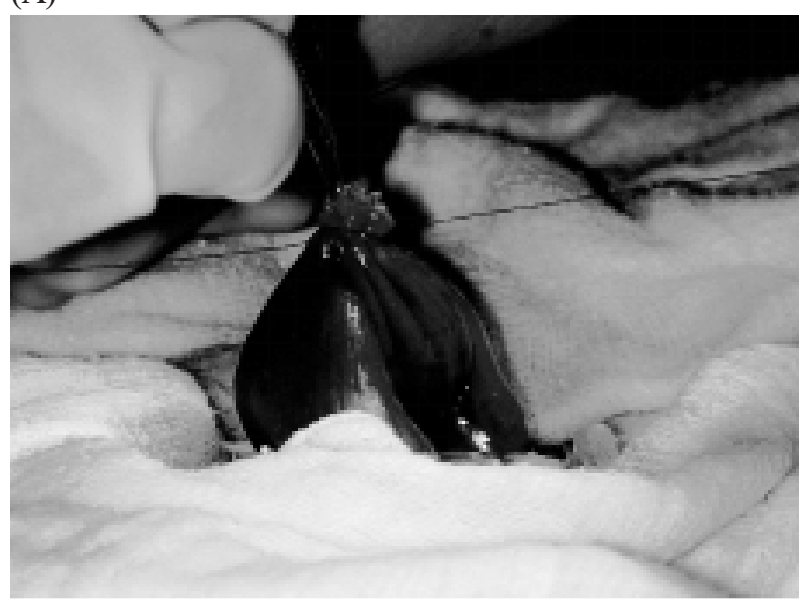

uterina. Este fato também tornava muito difícil a visibilização da dura-máter, por vezes levando a uma lesão não intencional da artéria e ou da medula espinhal. Exteriorizando apenas o rabo do feto, como descrevemos, há uma grande redução na tendência fetal de "descer", reduzindo a manipulação necessária para manter a área de interesse no lugar, diminuindo a duração do procedimento.

Ao operar em campos cirúrgicos maiores, dissecamos todas as camadas anatômicas até nosso objetivo ser alcançado, usando afastadores para expor e manter planos superficiais fora do caminho. Em um campo cirúrgico tão pequeno, uma dissecção/exposição adequada dos planos é muito difícil, e a visibilização da medula antes de produzir uma lesão não intencional era praticamente inevitável. À aplicação, uma suave pressão (como descrito) sobre a inserção das patas traseiras fetais, no momento da incisão da lâmina, facilitou a muito visibilização da medula espinhal. Usando esta técnica, quando a lâmina é cortada, a medula quase "salta para fora" do canal vertebral, reduzindo o risco de um dano "acidental" da medula.

Outra diferença importante de nossa técnica é o tipo de reparo miometrial, aplicado antes de sua abertura. Calvano $^{11}$ e Housley ${ }^{12}$ aplicaram uma ou duas suturas em bolsa, que depois da operação eram amarradas para fechar a cavidade amniótica e o miométrio (Figura 3A). Esta sutura parecia envolver maior quantidade de miometrio na sutura, reduzindo o tamanho da cavidade amniótica restante. Optou-se por abrir o miometrio aplicando somente dois pontos de reparo, um deles, ao final do procedimento, foi usado para executar uma sutura contínua "invaginante" quando do fechamento da incisão (Figura 3B), evitando o vazamento do líquido amniótico.

(B)

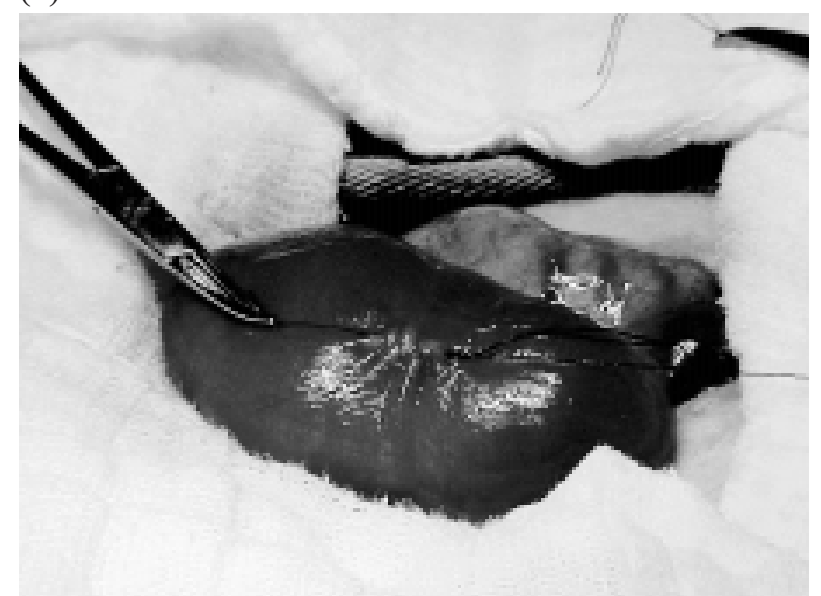

FIGURA 3 - Aspectos de duas diferentes suturas de fechamento aplicadas ao miométrio. (A) Sutura em bolsa, usada no projeto piloto. (B) Técnica de sutura invaginante, utilizada no estudo. Observar o aspecto "isquêmico" e a quantidade de miométrio envolvido na sutura em bolsa quando comparado à sutura invaginante 
Comparando a sobrevida etre os grupos I e II, seria esperado que a sobrevida no grupo em que a correção era tentada (grupo I) fosse mais baixa que no grupo não submetido a correção (grupo II), porque os fetos eram muito mais manipulados no primeiro caso. Os $73,3 \%$ de taxa de sobrevida encontrada neste grupo reforçam a impressão que esta técnica modificada pode ser melhor que as anteriormente descritas ${ }^{11,12}$.

O óbito fetal pós-operatório pode ter numerosas causas: comprometimento vascular placenta/umbilical, sangramento excessivo, perda de líquido amniótico e lesão medular. Dentre as modificações introduzidas por esta nova técnica, não foi possível estabelecer qual foi o ponto mais importante na melhora da sobrevida, mas acredita-se que a melhor exposição dos planos (reduzindo o dano à medula espinhal) e a técnica de fechamento do miométrio foram os principais responsáveis pela baixa de mortalidade fetal.

\section{Conclusão}

A técnica modificada apresenta sobrevida fetal satisfatória, no modelo experimental de criação de defeito espinhal em fetos.

\section{Referências}

1. Bruner JP, Tulipan N, Paschall RL, et al. Fetal surgery for MMC and the incidente of shunt-dependent hydrocephalus. J A Med Assoc 1999; 1819-25.

2. Sutton LN, Adzick NS, Bilanuik LT et al. Improvement in hindbrain herniation demonstrated by serial fetal MRI following fetal surgery for myelomeningocele. J A Med Assoc 1999; 282:1826-31.
3. Paek BW, Farmer D, Wilkinson C, Craig TA, Peacock W, Harrison MR, Jennings RW. Hindbrain herniation develops in surgically created myelomeningocele but is absent after repair in fetal lambs. Am J Obstet Gynecol 2000;183:1119-23.

4. Michejda M. Intrauterine treatment of spina bifida: primate model. Z Kinderchir 1984; 39:259-61.

5. Heffez DS, Aryanpur J, Hutchins, Freeman JM. The paralysis associated with myelomeningocele: clinical and experimental data implicating a preventable spinal cord injury. Neurosurgery 1990; 26:987-92.

6. Meuli M, Meuli-Simmen C, Hutchins GM, Seller MJ, Harrison MR, Adzick NS. The spinal cord lesion in human fetuses with myelomeningocele: implications for fetal surgery. J Pediatr Surg 1997; 32:448-52

7. Meuli M, Meuli-Simmen C, Yingling CD, Hutchins GM, Timmel GB, Harrison MR, Adzick NS. In utero repair of experimental myelomeningocele saves neurological function at birth. J Pediatr Surg 1996; 31:397-402.

8. Meuli M, Meuli-Simmen C, Yingling CD, Hutchins GM, Hoffman KM, Harrison MR, Adzick NS. Creation of myelomeningocele in utero: a model of functional damage from spinal cord exposure in fetal sheep. J Pediatr Surg 1995; 30:1028-32.

9. Meuli-Simmen C, Meuli M, Hutchins GM, Harrison MR, Buncke HJ, Sullivan KM, Adzick NS. Fetal reconstructive surgery: Experimental use of the latissimus dorsi flap to correct myelomeningocele in utero. Plast Reconstr Surg 1995; 96:1007-1011

10. Bruner JP, Richards WO, Tulipan NB, Arney TL. Endoscopic coverage of fetal myelomeningocele in utero. Am J Obstet Gynecol 1999; 180:153-8.

11. Calvano CJ; Reddy PP; Moran ME; Boyle KE; Housley HT; Jennings RW; Harrison MR; Mandell J. Initial studies of holmium:YAG laser creation of spinal defects in fetal rabbits: model for urologic effects of myelomeningocele. J Endourol 1998; 12:199-203.

12. Housley HT, Graf JL, Lipshultz GS, Calvano CJ, Harrison MR, Farmer DL, Jennings RW. Creation of myelomeningocele in the fetal rabbit. Fetal Diagn Ther 2000;15:272-9.

13. Fauza DO, Tannuri U, Ayoub AA, Capelozzi VL, Saldiva PH, Maksoud JG. Surgically produced congenital diaphragmatic hernia in fetal rabbits. J Pediatr Surg 1994;29:882-6 
$\overline{\text { Pedreira DAL, Valente P, Abou-Jamra RC, Pelarigo CL, Silva LM, Goldenberg S. A different technique }}$ to create of a myelomeningocele-like defect in the fetal rabbit. Acta Cir Bras [serial online] 2003 Mar-Apr;18(2). Available from URL: http://www.scielo.br/acb.

ABSTRACT - Purpose: To evaluate fetal survival rate using a modified technique to surgically create a "myelomeningocele-like" defect in the rabbit model. Methods: Six white New Zealand rabbits had a spinal defect created in their fetuses at 23 days of gestation. The defect was created in 19 fetuses and an attempt to correct it was made in 15 cases (group I), 4 fetuses where left without correction (group II)At 30 days of gestation, fetuses were harvested for anathomo-pathologic evaluation. Results: At 30 days, a "myelomeningocele-like" defect was present in all surviving fetuses. The total survival rate was 73,68\% (14/19), 11 fetus survived in group I and 3 in group II. Conclusion: The technique modifications including fetal positioning and exposure of its back prior to the incision of the lamina associated to a different technique for miometrium closure offers an alternative and probably safer method to surgically create a spinal defect in the fetal rabbit.

KEY WORDS - Fetal surgery. Meningomyelocele. Spinal dysraphism. Rabbit. Prenatal. Disease models, animal.

Conflito de interesse: nenhum Fonte de financiamento: FAPESP

Correspondência:

Denise Araújo Lapa Pedreira

Rua Bagé, 163/182

04012-140 São Paulo - SP

Tel/Fax: (11)5572-2033

denise_lapa@einstein.br

wdpedreira@uol.com.br

Data do recebimento: 08/01/2003

Data da revisão: 18/01/2003

Data da aprovação: 25/01/2003 